\title{
Clinical Reasoning: Rapid progression of reversible cognitive impairment in an 80-year-old man
}

Walid Bouthour, MD, Lukas Sveikata, MD, Maria Isabel Vargas, MD, Johannes Alexander Lobrinus, MD, and Emmanuel Carrera, MD

Neurology ${ }^{\circledR}$ 2018;91:1109-1113. doi:10.1212/WNL.0000000000006647

\author{
Correspondence \\ Dr. Carrera \\ emmanuel.carrera@ \\ hcuge.ch
}

\section{Section 1}

An 80-year-old right-handed man was accompanied by his wife to the neurology clinic for difficulties in performing activities of daily living. He was reported to struggle using cutlery and remembering how to dial a phone number. His wife had noticed changes in his speech, which had become incomprehensible. Symptom onset was insidious, with progressive worsening over a couple of weeks. The patient himself had no complaint. His medical history included an episode of transient vertical diplopia 2 months prior to admission, diagnosed as a TIA in the midbrain. Brain MRI at the time of TIA showed no acute lesion but moderate vascular white matter disease (WMD), related to arterial hypertension and type II diabetes, and 11 lobar microbleeds (bilateral temporal, parietal, and frontal).

Neurologic examination at the time of the current admission revealed right homonymous hemianopia, production of jargon and impaired comprehension suggestive of Wernicke aphasia, ideational apraxia, executive dysfunction, and anosognosia. The rest of the physical examination was normal. Episodes of paroxysmal atrial fibrillation were detected during the patient's hospital stay.

\section{Questions for consideration:}

1. Can you relate the clinical picture to a single lesion?

2. What is your differential diagnosis at this stage?

\section{GO TO SECTION 2}

From the Departments of Neurology (W.B., L.S., E.C.), Neuroradiology (M.I.V.), and Clinical Pathology (J.A.L.), Geneva University Hospital and University of Geneva, Switzerland. Go to Neurology.org/N for full disclosures. Funding information and disclosures deemed relevant by the authors, if any, are provided at the end of the article. 


\section{Section 2}

Both Wernicke aphasia and ideational apraxia suggest a dysfunction in the left posterior hemisphere; more specifically in temporoparietal associative cortices for the Wernicke aphasia and in the supramarginal gyrus for ideational apraxia. Right homonymous hemianopia indicates involvement of the left visual pathways. Although executive functions are widely distributed throughout the brain, executive dysfunction is classically associated with pathologies of prefrontal brain regions. Anosognosia is the result of a lesion in the right temporoparietal lobe. The symptoms and signs suggest widespread bihemispheric dysfunction predominantly affecting the posterior part of the left hemisphere.

In our patient, the rapidly progressive evolution of cognitive decline points towards several etiologies including vascular, infectious, and inflammatory processes. ${ }^{1}$ Vascular causes should be considered because of the history of chronic arterial hypertension, possible TIA, and WMD. However, the absence of an acute or stepwise evolution does not favor a vascular etiology. Among infectious causes, herpes simplex virus encephalitis was ruled out because of the subacute evolution of the disease and the absence of fever or seizures. Neurosyphilis and HIV dementia have a more indolent evolution, and the patient had no sexual risk factors. There was no tick bite or erythema migrans history to support Lyme disease. Voltagegated potassium channel (VGKC), NMDA receptor, and other antibody-related autoimmune encephalitis are frequently associated with myoclonus or seizures. Paraneoplastic autoimmune encephalitis may also cause rapidly progressive cognitive and neuropsychiatric symptoms. Other inflammatory causes include cerebral vasculitis, which could present with subacute cognitive impairment. Inflammatory cerebral amyloid angiopathy is a rare and newly recognized entity that may cause subacute dementia in predominantly older patients. Creutzfeldt-Jacob disease can present with rapid cognitive decline, sometimes associated with visual field defects, myoclonus, or seizures. In the absence of preexisting cognitive decline reported by the family, Alzheimer disease and frontotemporal disease were considered less likely. As for tumor-related pathologies, lymphoma typically presents with progressive cognitive decline, and brain metastases can cause rapidly progressive cognitive symptoms.

\section{Question for consideration:}

1. Which ancillary tests would you perform to make the diagnosis?

GO TO SECTION 3 


\section{Section 3}

Brain MRI, FSE T2-weighted sequence, showed bilateral and asymmetric cortical and subcortical hyperintense lesions in the left parietal, temporal, and occipital lobes with a discrete mass effect on adjacent structures (figure 1). Axial T2*weighted images showed the same lobar microbleeds as described previously. There was no superficial hemosiderosis or signs of past intracerebral hemorrhage. Gadolinium-enhanced T1-weighted sequence showed no parenchymal or meningeal enhancement. Magnetic resonance spectroscopy revealed a decrease of the $\mathrm{N}$-acetylaspartate/choline ratio, but no characteristic pattern of brain tumor.

Laboratory investigations, including blood electrolytes, C-reactive protein, erythrocyte sedimentation rate, liver function, and blood cell count (particularly white blood cells, $5.9 \mathrm{~g} / \mathrm{L}$, normal 4-11), were normal. HIV, Treponema pallidum hemagglutination, and Lyme disease screening was negative. VGKC and NMDA antibodies, antinuclear antibodies, and anti-double-stranded DNA antibodies were also negative. Characterization of $A P O E$ gene showed a heterozygous APOE with $\varepsilon 4 / \varepsilon 3$ alleles. Finally, CSF examination revealed increased protein concentration $(1.45 \mathrm{~g} / \mathrm{L}$, normal $<0.45$ ), normal cell count $(<1 \mathrm{M} / \mathrm{L}$, normal $0-5)$, normal glucose level $(4.0 \mathrm{mmol} / \mathrm{L}$, normal $2.8-4.0)$, no oligoclonal bands, and no abnormal cells. The subacute evolution of the clinical picture and apraxia should prompt a thorough assessment for Creutzfeldt-Jacob disease. However, the MRI did not support this hypothesis.

\section{Questions for consideration:}

1. What is your diagnosis at this stage?

2. How would you confirm the diagnosis?
Figure 1 Brain MRI slices before admission, upon admission, and during follow-up

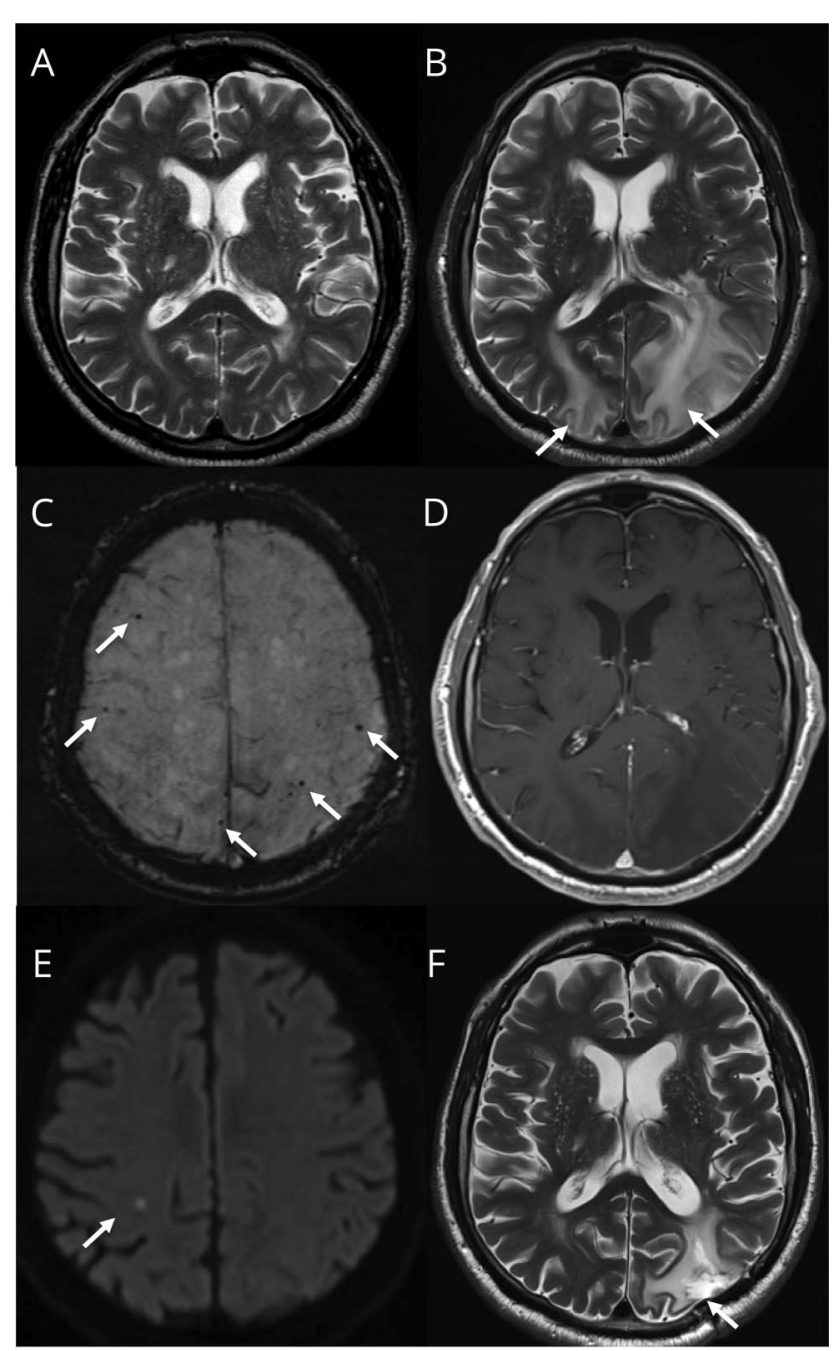

(A) No lesion on axial fast spin echo (FSE) T2-weighted imaging sequence after the transient focal episode, 2 months prior to admission. (B) Axial FSET2 MRI upon admission shows high signal on both parieto-occipital lobes (arrows) and (C) axial T2*-weighted sequence shows several lobar microbleeds (arrows). (D) Axial, gadolinium-enhanced T1-weighted sequence shows no enhancement. (E) A small frontal cortico-subcortical ischemic lesion is revealed by diffusion-weighted imaging (arrow) on a follow-up MRI 4 months after onset. (F) Final follow-up axial FSE-T2 MRI shows regression of hyperintensities (arrow).

\section{GO TO SECTION 4}




\section{Section 4}

The clinical picture, imaging, CSF, and blood workup are suggestive of inflammatory cerebral amyloid angiopathy (CAA-I). The patient also carried the APOE $\varepsilon 4$ allele, which increases the risks of $\beta$-amyloid peptide deposition and CAArelated hemorrhage compared to carriers of the common $\varepsilon 3$ alleles.

To confirm the diagnosis, we performed a brain biopsy in the left posterior parietal lobe including cortical and subcortical brain tissue. Cerebral biopsy showed transmural inflammatory infiltrates and amyloid deposition within vessels, and perivascular inflammatory infiltrates with reactive astrogliosis and microglial activation (figure 2). This provides evidence for 2 variants of CAA-I. The transmural inflammatory infiltrate is a destructive vasculitic process that characterizes $A \beta$-related angiitis (ABRA), and perivascular inflammatory infiltrates are characteristic of CAA-related inflammation (CAA-ri). ${ }^{2}$ Both subtypes can coexist in patients with CAA- $\mathrm{I}^{3}$ and respond to immunosuppressive treatment, but it is not established whether such pathologic subtypes translate into separate entities that require different therapeutic management. ${ }^{2}$

\section{Questions for consideration:}

1. Which treatment would you start?

2. How would you relate the history of transient symptoms (vertical diplopia) to the diagnosis of CAA-I?

Figure 2 Cortical brain biopsy in the left parietal lobe

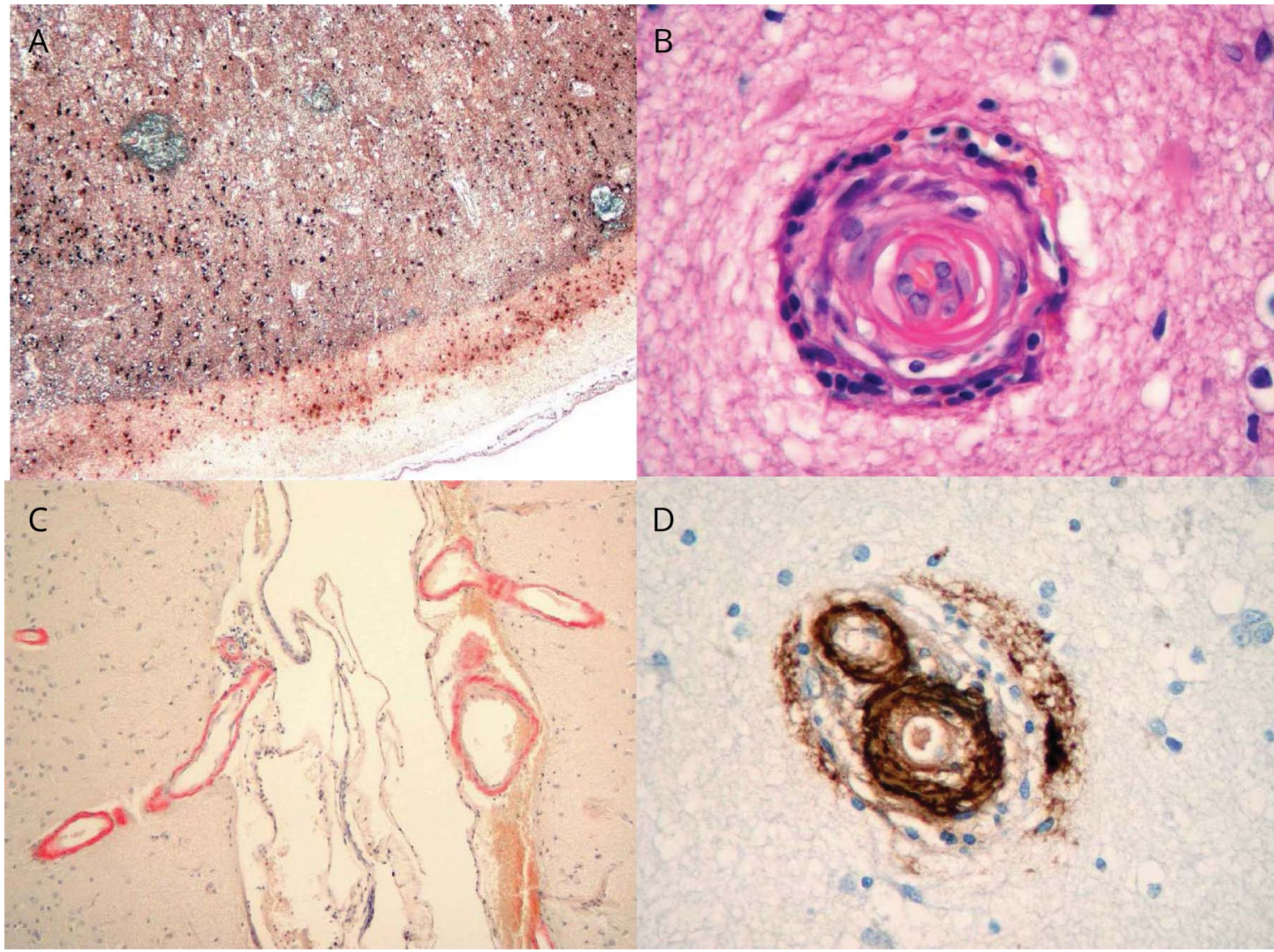

(A) NeuN immunostaining shows cortical edema and loss of neurons (magnification $\times 100$ ). (B) Hematoxylin \& eosin staining shows eosinophilic deposits within the wall of a cortical vessel, lumen stenosis, and perivascular inflammatory infiltrates (magnification $\times 400)$. (C) Congo red staining shows amyloid deposits within the wall of cerebral and meningeal vessels (magnification $\times 40$ ). (D) $\beta$-Amyloid immunostaining shows $A \beta$ deposition within the walls of a cortical vessel (magnification $\times 400)$

\section{GO TO SECTION 5}




\section{Section 5}

We initiated immunosuppressive treatment, consisting of oral prednisone, $1 \mathrm{mg} / \mathrm{kg}$ a day, tapered over 3 months, with a good initial clinical and radiologic evolution. However, 1 month after the end of immunosuppression (i.e., 4 months after diagnosis of CAA-I), the patient experienced rapid worsening of cognitive functions. MRI at the time showed the recurrence of the same initial hyperintensity at the same location, and an acute, asymptomatic diffusion-weighted imaging (DWI) punctiform ischemia in the right subcortical parietal lobe. We reinitiated oral prednisone and introduced oral azathioprine with a favorable clinical evolution. Follow-up MRI did not show recurrence of the inflammatory findings of CAA, but 6 new lobar microbleeds were reported over the 36-month follow-up.

Regarding the episode of regressive vertical diplopia presented by our patient 2 months prior to admission, several hypotheses may exist. Transient focal neurological episodes or "amyloid spells," defined as short, recurrent, stereotyped, spreading neurologic symptoms, are frequently reported in patients with CAA and are often related to superficial hemosiderosis or subarachnoid hemorrhage. ${ }^{3}$ A TIA is also possible given the age, cardiovascular risk factors, and detection of episodes of atrial fibrillation. Interestingly, small asymptomatic DWI ischemic lesions have been described recently in patients with CAA over the course of the disease. ${ }^{3,4}$

\section{Discussion}

The diagnosis of CAA-I was established based on (1) cerebral MRI showing rapid onset leukoencephalopathy and edema; (2) CSF analysis displaying mildly elevated protein concentration, but no pleiocytosis; and (3) neuropathology demonstrating perivascular inflammation associated with amyloid deposits in cortical vessels and transmural and perivascular inflammation. Before confirmation with cerebral biopsy, our patient met the diagnostic criteria of probable CAA-I: (1) age $>40$ years; (2) clinical symptoms, such as headaches or cognitive disturbances, not directly attributable to intracerebral hemorrhage; (3) asymmetric WMD patterns that extend to the immediately subcortical area; and (4) presence of cortico-subcortical hemorrhagic lesions. ${ }^{5}$

The inflammatory variants of CAA have gained a great amount of interest lately because of their increased incidence in the elderly population. Unlike classical CAA, inflammatory variants do not usually present with intracerebral hemorrhage, and the symptoms may be reversed by immunomodulatory therapy. ${ }^{4}$ However, CAA without inflammation can also exhibit infiltrative white matter processes. ${ }^{6}$ In order to avoid undue immunosuppressive treatment and the side effects thereof, we carried out a brain biopsy to look for vascular inflammation that characterizes CAA-I.
An interesting point raised by this case is the speed of the clinical and radiologic course. The fact that lobar microbleeds were present before the onset of CAA-I supports the preexistence of CAA. The subsequent cognitive symptoms were more likely linked to the CAA-I, as supported by the clinical and radiologic features that progressed rapidly over 2 months. Based on repetitive MRIs and cerebral biopsy findings, our case illustrates that the course of CAA-I may be subacute and clearly distinct from the more progressive evolution of the noninflammatory form of CAA. ${ }^{6}$

A second interesting point is the management of transient neurologic symptoms and the ischemic lesion on DWI attributed to CAA. ${ }^{7,8}$ Our patient had CAA, but also cardiovascular disease risks factors (diabetes, hypertension) and episodes of atrial fibrillation. The presence of $\geq 5$ cerebral microbleeds carries a major risk of intracerebral hemorrhage with anticoagulant use (odds ratio 5.50 or $2.8 \% / y$ ). ${ }^{9}$ Therefore, we opted for a percutaneous left atrial appendage closure, although it is not known if the bleeding risk is increased to the same extent in the inflammatory form of CAA.

\section{Author contributions}

Research project conception: E. Carrera. Research project execution: W. Bouthour, L. Sveikata. Writing of the first draft: W. Bouthour, L. Sveikata. Specialized material (radiology, pathology): M.I. Vargas, J.A. Lobrinus. Review and critique: E. Carrera, M.I. Vargas, J.A. Lobrinus.

\section{Study funding}

No targeted funding reported.

\section{Disclosure}

The authors report no disclosures relevant to the manuscript. Go to Neurology.org/N for full disclosures.

\section{References}

1. Paterson RW, Takada LT, Geschwind MD. Diagnosis and treatment of rapidly progressive dementias. Neurol Clin Pract 2012;2:187-200.

2. Salvarani C, Hunder GG, Morris JM, Brown RD, Christianson T, Giannini C. A $\beta$ related angiitis: comparison with CAA without inflammation and primary CNS vasculitis. Neurology 2013;81:1596-1603.

3. Charidimou A, Peeters A, Fox Z, et al. Spectrum of transient focal neurological episodes in cerebral amyloid angiopathy: multicentre magnetic resonance imaging cohort study and meta-analysis. Stroke 2012;43:2324-2330.

4. Corovic A, Kelly S, Markus HS. Cerebral amyloid angiopathy associated with inflammation: a systematic review of clinical and imaging features and outcome. Int $\mathrm{J}$ Stroke 2017;73:1-11.

5. Auriel E, Charidimou A, Gurol ME, et al. Validation of clinicoradiological criteria for the diagnosis of cerebral amyloid angiopathy-related inflammation. JAMA Neurol 2015;73:197-202.

6. Salvarani C, Morris JM, Giannini C, Brown RD Jr, Christianson T, Hunder GG. Imaging findings of cerebral amyloid angiopathy, A $\beta$-related angiitis (ABRA), and cerebral amyloid angiopathy-related inflammation. Medicine 2016;95: e3613-e3617.

7. Kimberly WT, Gilson A, Rost NS, et al. Silent ischemic infarcts are associated with hemorrhage burden in cerebral amyloid angiopathy. Neurology 2009;72: 1230-1235.

8. van Veluw SJ, Lauer A, Charidimou A, et al. Evolution of DWI lesions in cerebral amyloid angiopathy. Neurology 2017;89:2136-2142.

9. Charidimou A, Karayiannis C, Song TJ, et al. Brain microbleeds, anticoagulation, and hemorrhage risk. Neurology 2017;89:2317-2326. 


\section{Neurology}

\section{Clinical Reasoning: Rapid progression of reversible cognitive impairment in an 80-year-old man \\ Walid Bouthour, Lukas Sveikata, Maria Isabel Vargas, et al. Neurology 2018;91;1109-1113 \\ DOI 10.1212/WNL.0000000000006647}

\section{This information is current as of December 10, 2018}

\section{Updated Information \& Services}

References

Citations

Subspecialty Collections

Permissions \& Licensing

\section{Reprints}

including high resolution figures, can be found at: http://n.neurology.org/content/91/24/1109.full

This article cites 9 articles, 6 of which you can access for free at: http://n.neurology.org/content/91/24/1109.full\#ref-list-1

This article has been cited by 1 HighWire-hosted articles: http://n.neurology.org/content/91/24/1109.full\#\#otherarticles

This article, along with others on similar topics, appears in the following collection(s):

\section{Cognitive aging}

http://n.neurology.org/cgi/collection/cognitive_aging

MRI

http://n.neurology.org/cgi/collection/mri

Other cerebrovascular disease/ Stroke

http://n.neurology.org/cgi/collection/other_cerebrovascular_disease_st roke

Information about reproducing this article in parts (figures,tables) or in its entirety can be found online at:

http://www.neurology.org/about/about_the_journal\#permissions

Information about ordering reprints can be found online:

http://n.neurology.org/subscribers/advertise

Neurology ${ }^{\circledR}$ is the official journal of the American Academy of Neurology. Published continuously since 1951, it is now a weekly with 48 issues per year. Copyright (O) 2018 American Academy of Neurology. All rights reserved. Print ISSN: 0028-3878. Online ISSN: 1526-632X.

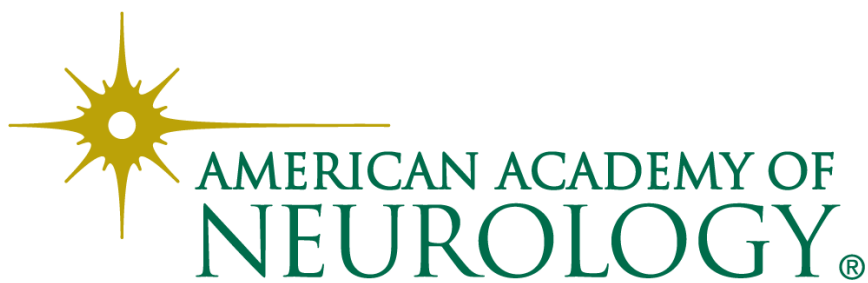

